\title{
ANOS POTENCIAIS DE VIDA PERDIDOS NO BRASIL EM DECORRÊNCIA DA DENGUE: IMPACTO SOCIOECONÔMICO
}

\author{
YEARS POTENTIALS OF LIFE LOST IN BRAZIL DUE TO DENGUE'S: \\ SOCIOECONOMIC IMPACT
}

\author{
Thales Lemos Pimentel ${ }^{1}$, Wesley Abyjaude ${ }^{2}$, Eduardo Frias Corrêa Oliveira ${ }^{3}$, Karen \\ Helen Martins Canazart ${ }^{4}$, João Vitor Andrade ${ }^{5}$
}

\begin{abstract}
${ }^{1}$ Graduando em Medicina na Universidade Federal de Viçosa - UFV. Viçosa (MG), Brasil. ORCiD: https://orcid.org/0000-0002-1413-4398

${ }^{2}$ Graduando em Enfermagem na Universidade Federal de Viçosa - UFV. Viçosa (MG), Brasil. ORCiD: https://orcid.org/0000-0002-6384-3270

${ }^{3}$ Graduando em Enfermagem na Universidade Federal de Viçosa - UFV. Viçosa (MG), Brasil. ORCiD: https://orcid.org/0000-0002-9616-2439

${ }^{4}$ Graduanda em Enfermagem na Universidade Federal de Viçosa - UFV. Viçosa (MG), Brasil. ORCiD: https://orcid.org/0000-0002-1922-7104

${ }^{5}$ Enfermeiro. Especializando em Enfermagem em Saúde Mental e Psiquiátrica na Universidade de Saúde Paulo. São Paulo (SP), Brasil. ORCiD: : https://orcid.org/0000-0003-3729-501X

Endereço para correspondência: thales.pimentel@ufv.br
\end{abstract}

Declaração de conflitos de interesse: não há.

\section{RESUMO}

OBJETIVO: o presente objetiva caracterizar o impacto dos óbitos em decorrência da dengue no Brasil nos últimos dez anos. MÉTODOS: para estimativa do impacto socioeconômico, utilizou-se a variável Anos Potenciais de Vida Perdidos (APVP), estabelecendo-se como limite para o cálculo a idade de 75 anos. Foram selecionados os dados referentes aos óbitos por dengue no Brasil no período de 2009 a 2018. RESULTADOS: o número de óbitos notificados de indivíduos até 75 anos, em decorrência da dengue foi de 4.295, totalizando 144.301 APVP. A dengue, apesar de ser uma doença negligenciada, gera perdas econômicas elevadas ao país, as maiores dentre o Hemisfério Ocidental. CONCLUSÃO: destarte, é imprescindível aumentar os investimentos nessa temática, de forma a permitir profilaxia e manejo adequados dessa condição, para que seja possível fortalecer estratégias com potencial para minimização da morbimortalidade pela doença.

Palavras-chave: Dengue; Anos Potenciais de Vida Perdidos; Mortalidade Prematura; Saúde Pública. 


\begin{abstract}
OBJECTIVE: this study aims to characterize the impact of deaths due to dengue in Brazil in the last ten years. METHODS: to estimate the socioeconomic impact, the variable Potential Years of Life Lost (APVP) was used, establishing the age limit of 75 as the calculation limit. Data on deaths from dengue in Brazil in the period from 2009 to 2018 were selected. The number of reported deaths of individuals up to 75 years of age, due to dengue was 4,295, totaling 144,301 APVP. Dengue, despite being a neglected disease, generates high economic losses to the country, the greatest among the Western Hemisphere. CONCLUSION: thus, it is essential to increase investments in this topic, in order to allow prophylaxis and adequate management of this condition, so that it is possible to strengthen strategies with the potential to minimize morbidity and mortality from the disease.
\end{abstract}

Keywords: Dengue; Potential Years of Life Lost; Premature Mortality; Public Health.

\title{
INTRODUÇÃO
}

A dengue é uma virose sistêmica aguda, causada pelo vírus dengue, da família Flaviviridae e gênero Flavivirus, com quatro sorotipos distintos: Den 1, Den 2, Den 3 e Den $4^{1}$. Cada sorotipo, por sua vez, possui genótipos que denotam sua grande variabilidade genética e, consequentemente, diferentes potenciais de induzir lesão nos indivíduos infectados ${ }^{1,2}$. O vírus está inserido no grande grupo dos arbovírus, ou seja, vírus transmitidos por artrópodes, nesse caso, insetos hematófagos (a fêmea) do gênero Aedes ${ }^{3}$.

Faz-se referência à dengue no Brasil desde 1846, entretanto, tal agravo só adquiriu importância epidemiológica a partir de 1986, quando irrompeu a epidemia no estado do Rio de Janeiro e a circulação do sorotipo 1 , que logo alcançou a região Nordeste ${ }^{2}$. Dessa forma, a dengue se torna endêmica no Brasil, intercalando-se epidemias, geralmente associadas à introdução de novos sorotipos, em áreas anteriormente indenes ${ }^{2,3}$.

Caracteriza-se, na forma clássica, denominada febre dengue ou dengue clássico, por quadro febril abrupto, algias variadas, exantema pruriginoso ou não, e manifestação hemorrágica ${ }^{1}$. Na maioria das vezes, o período sintomático não ultrapassa 10 dias, destaca-se ainda que quase $100 \%$ dos casos evoluem para a cura ${ }^{1,3}$. Uma apresentação grave, pouco 
frequente, é a temida febre hemorrágica do dengue, cuja importância se deve ao aparecimento em razoável número durante epidemias e ao potencial risco de óbito ${ }^{1}$.

Nos últimos 50 anos sua incidência aumentou 30 vezes, expondo 2,5 bilhões de pessoas à infecção, em especial da Ásia, América Latina, Caribe e África ${ }^{3}$. Estima-se 50 milhões de novos casos a cada ano e na América Latina há epidemias cíclicas a cada 2-5 anos ${ }^{1}$. Em 2019, foram notificados 1.544 .987 casos prováveis de dengue no Brasil, a uma taxa de incidência de 735,2 casos por 100 mil habitantes ${ }^{4}$. Até o início do mês de maio de 2020, o país já havia registrado 676.928 casos prováveis de dengue, uma proporção maior do que para o mesmo período de tempo dos anos epidêmicos de 2015 e $2019^{2}$.

Considerada uma entidade com ampla possibilidade de apresentação clínica, desde casos oligossintomáticos, passando pela forma clássica, até a forma hemorrágica do dengue com ou sem choque, os fluxogramas para manejo clínico deste agravo principiam-se na classificação do paciente em um dos estágios da doença de acordo com sua gravidade ${ }^{1,2}$. Em casos mais leves, são prescritos repouso, hidratação vigorosa e sintomáticos caso haja necessidade, como analgésicos e antieméticos ${ }^{1}$. Para quadros mais graves, a reposição volêmica é feita de modo mais intenso, por via intravenosa e compreendendo as fases de expansão e manutenção, e em alguns casos, uso de expansores plasmáticos e coloides sintéticos ${ }^{1}$.

A despeito da elevada prevalência da dengue tanto em território nacional quanto internacional, tal agravo é reconhecido como uma doença negligenciada, ou seja, há um baixo interesse da indústria farmacêutica pela temática, justificado pelo reduzido potencial de retorno lucrativo para a indústria, uma vez que a população atingida é de baixa renda e presente, em sua maioria, nos países em desenvolvimento ${ }^{3,5}$.

Nesse sentido, na inexistência de vacinas e fármacos para a profilaxia e o manejo adequado da dengue, a enfermidade persiste em ser responsável por um número considerável de óbitos, os quais resultam em impacto socioeconômico para o Brasil, tanto pelos gastos de 
internação dos pacientes quanto pelos anos potenciais de vida perdidos (APVP). Neste sentido, o presente estudo tem por objetivo qualificar o impacto dos óbitos ocorridos no Brasil em decorrência da dengue, utilizando o indicar APVP.

\section{MÉTODOS}

O presente caracteriza-se como um estudo quantitativo, conduzido por dados secundários alocados no Sistema de Informação sobre Mortalidade (SIM), do Ministério da Saúde e disponíveis por meio do Departamento de Informática do Sistema Único de Saúde DATASUS $^{4}$. As variáveis coletadas foram: ano do óbito, sexo, região, grupo etário e causa de óbito, sendo esta baseada nos códigos A90 e A91 da Classificação Internacional de Doenças e Problemas Relacionados à Saúde $-10^{\mathrm{a}}$ revisão ${ }^{6}$. Para o cálculo dos APVP, foi utilizada a técnica de Romeder e Whinnie, que estabelece o limite para o cálculo considerando a vida média da população ${ }^{7}$, que em decorrência da expectativa de vida dos brasileiros, a idade adotada foi de $75 \operatorname{anos}^{8,9}$

Para a obtenção do número de APVP foi realizada a distribuição dos óbitos por agrupamentos de idade. Em seguida, multiplicou-se o número de óbitos em cada intervalo de idade pelo número de anos restantes para atingir a idade limite de 75 anos ${ }^{7}$. Essa diferença foi obtida a partir do ponto médio de cada faixa etária. A somatória dos resultados desses produtos forneceu o total de APVP, valor que representa o número estimado de perdas de anos para uma causa específica de morte, nesse estudo em questão, a dengue.

\section{RESULTADOS}

O quantitativo de óbitos notificados no SIM, de indivíduos abaixo de 1 ano até 74 anos de idade, em decorrência da dengue nos anos de 2009 a 2018 foi de 4.295 , o que totaliza 144.301 APVP, conforme Tabela 1. 
Em relação aos óbitos na idade economicamente ativa (faixa etária de 15 a 64 anos), estes representam 69,01\% e totalizam 97.117 APVP (região hachurada na Tabela 1).

Tabela 1 - Quantitativo de óbitos e APVP por dengue no Brasil no período de 2009 a 2018, por faixa etária.

\begin{tabular}{ccc}
\hline Faixa etária & Número de óbitos & Quantitativo dos APVP \\
\hline Abaixo de 1 ano & 117 & 8717 \\
1 a 4 anos & 119 & 8627 \\
5 a 9 anos & 198 & 13464 \\
10 a 14 anos & 200 & 12600 \\
15 a 19 anos & 208 & 12064 \\
20 a 24 anos & 196 & 10388 \\
25 a 29 anos & 258 & 12384 \\
30 a 34 anos & 288 & 12384 \\
35 a 39 anos & 270 & 10260 \\
40 a 44 anos & 310 & 10230 \\
45 a 49 anos & 345 & 9660 \\
50 a 54 anos & 373 & 8579 \\
55 a 59 anos & 372 & 6696 \\
60 a 64 anos & 344 & 4472 \\
65 a 69 anos & 337 & 2696 \\
70 a 74 anos & 360 & 1080 \\
Total & $\mathbf{4 2 9 5}$ & $\mathbf{1 4 4 . 3 0 1}$ \\
\hline
\end{tabular}

Fonte: Dados do presente estudo, 2020.

Em relação ao total geral dos óbitos, a taxa de mortalidade no sexo masculino foi mais elevada em relação ao sexo feminino. Destaca-se ainda que o número de óbitos foi diferente nas regiões, dados esses evidenciados na Figura 1. 
Figura 1 - Óbitos no Brasil em decorrência da dengue, por região no período de 2009 a 2018.

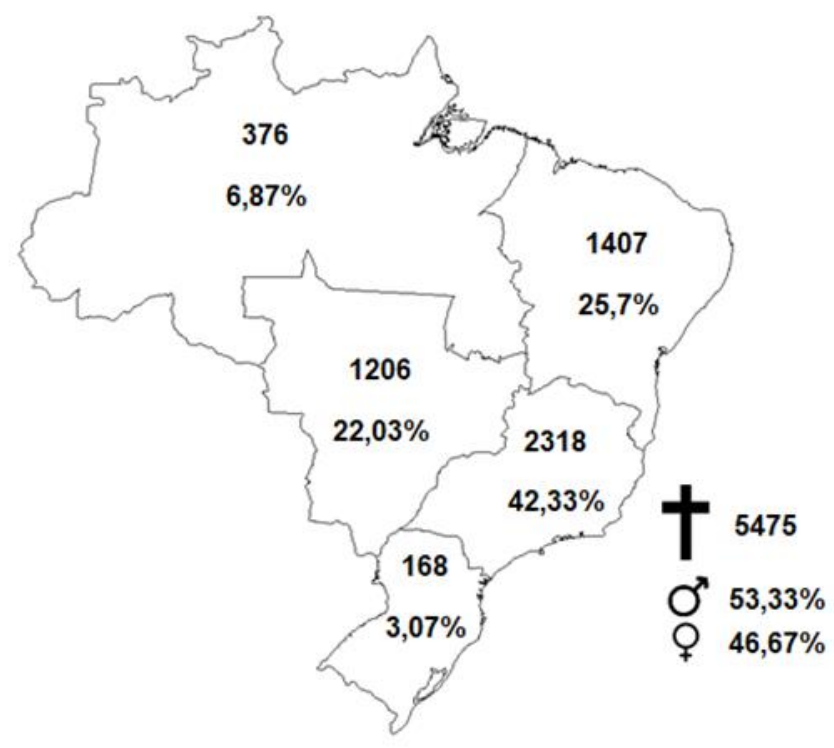

Fonte: Dados do presente estudo, 2020.

Quanto à evolução dos óbitos pela dengue no Brasil nos anos analisados, pode-se observar uma tendência à redução de tal número, principalmente no que tange ao período de 2015 a 2017.

Figura 2 - Óbitos no Brasil em decorrência da dengue, por ano no período de 2009 a 2018.

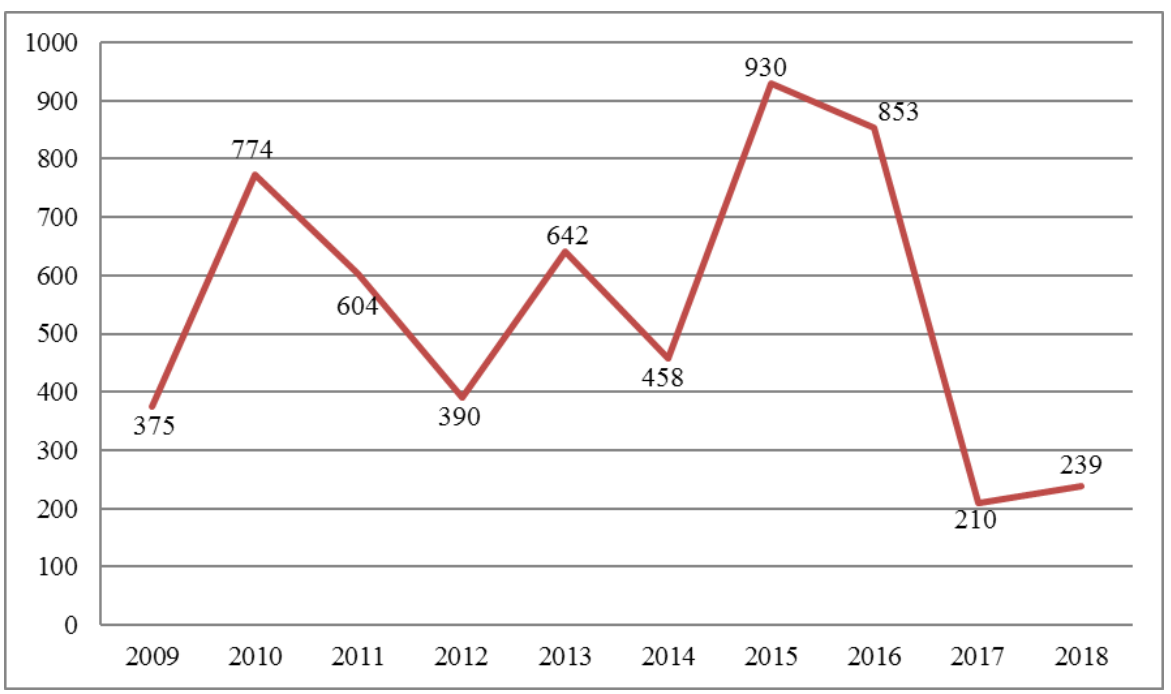

Fonte: Dados do presente estudo, 2020. 


\section{DISCUSSÃO}

De todos os países do Hemisfério Ocidental, o Brasil apresenta a maior perda econômica em decorrência da dengue ${ }^{2,3}$. No ano epidêmico de 2016, o investimento total no combate ao vetor atingiu o patamar de aproximadamente $\mathrm{R}$ \$ 1,5 bilhão no Brasil, além de $\mathrm{R}$ \$ 76,8 milhões reportados pelo governo federal para a aquisição de larvicidas e inseticidas, tendo os repasses federais se concentrado nos estados de São Paulo, Minas Gerais, Rio de Janeiro e Bahia². Outrossim, o país apresentou um gasto total de aproximadamente $\mathrm{R} \$ 175$ milhões no tratamento da dengue em 2016 e R\$ 114 milhões para manutenção dos centros especializados em reabilitação ${ }^{10}$.

Somam-se a esse cenário os custos indiretos por perda de produtividade por afastamentos relacionados à dengue, estimados em R\$ 293 milhões em 2016 segundo $^{10}$. Em adição aos custos indiretos, incluem-se os APVP supracitados, os quais se concentram entre a população em idade economicamente ativa, resultando em impacto socioeconômico de grande monta para o Brasil.

Uma pesquisa avaliou a eficácia de um sistema de Monitoramento Inteligente da Dengue (MID) para o controle do vetor, comparando-o com os procedimentos de diretrizes do Ministério da Saúde, o MID preveniu 27.191 novos casos de dengue em 21 cidades do estado de Minas Gerais, economizando aos cofres públicos um total de 364 mil dólares por custos diretos e 7 milhões de dólares por custos indiretos ${ }^{11}$. Destarte, tendo em vista o cenário de elevadas perdas econômicas pela dengue no país, torna-se imprescindível o investimento em estratégias mais eficazes de controle ao vetor e outras formas de profilaxia, como vacinas, além de fármacos, de forma a reduzir a perda de produtividade por afastamentos e APVP, além dos gastos médicos com internações de pacientes.

Em relação à frequência de óbitos por sexo, observou-se que a maioria das mortes por dengue notificadas no Brasil ocorreu em população do sexo masculino, representando 53,33\%, 
enquanto a população feminina totalizou 46,67\% dos óbitos. Todavia, em relação ao número de casos notificados, há uma prevalência desses entre o sexo feminino ${ }^{8,9}$. Tal fato é explicado pelas mulheres permanecerem mais tempo em suas residências que os homens e como a transmissão se faz principalmente no domicílio e peridomicílio, a diferença observada se dá devido à maior exposição ${ }^{9}$. Ademais, tanto o número de casos ser maior entre o sexo feminino quanto o número de óbitos ser maior entre o sexo masculino também se relacionam ao fato de as mulheres buscarem mais assistência médica que os homens ${ }^{12,13}$.

Quanto à distribuição dos óbitos por dengue de acordo com as regiões, os maiores quantitativos encontram-se nas regiões Sudeste e Nordeste, respectivamente, o que é condizente com o fato de serem as duas regiões mais populosas do país. A região Centro-Oeste, em adição, possui um número absoluto de óbitos próximo ao do Nordeste, fato que é um destaque negativo, tendo em vista se tratar de uma região com menor número de habitantes. A Região Norte, por sua vez, apresenta um histórico de poucos casos notificados, pois não obstante compreender 45,2\% do território nacional, trata-se de uma área com baixa densidade populacional, além de possuir menor quantitativo de centros urbanos que no restante do país ${ }^{14}$.

Chama atenção, entretanto, os números de óbitos na região Sul, visto que, apesar de apresentar uma população considerável, registra os menores quantitativos no país. De acordo com os dados coletados, a incidência dos casos de dengue na região Sul tende a ser menor em comparação às demais regiões. No ano epidêmico de 2015, por exemplo, a região foi responsável por apenas $3,07 \%$ do total das cinco macrorregiões ${ }^{8,9}$. Uma das possíveis explicações para tal distinção encontra-se no clima, tendo a região Sul estações bem definidas e temperaturas menores em relação às demais regiões do país ${ }^{15}$. Essas condições, embora não impeçam, desfavorecem a proliferação do Aedes aegypti.

Quanto à evolução dos óbitos por dengue no Brasil ao longo dos anos, pode-se verificar o caráter cíclico da doença, intercalando períodos de endemicidade com epidemicidade, como 
na epidemia ocorrida no país em 2015 e 2016, a qual sucederam-se dois anos de redução no número de óbitos (2017 e 2018) e então, desde 2019 encontra-se em curso uma nova epidemia.

\section{CONCLUSÃO}

No Brasil, a permanência da dengue como enfermidade endêmica é responsável por um número considerável de casos e óbitos, os quais geram grande impacto socioeconômico para o cenário nacional, conforme dados supracitados. Todavia, tal agravo é reconhecido como uma doença negligenciada, para a qual não há interesse da indústria farmacêutica e das esferas governamentais no desenvolvimento de vacinas, fármacos e mecanismos de controle do vetor. Nesse sentido, tendo em vista o Brasil possuir as maiores perdas econômicas em decorrência da dengue no Hemisfério Ocidental, é nítida a necessidade de se abandonar a visão de negligência e possibilitar maiores investimentos na temática, de forma a reduzir os futuros gastos na pasta e assim, fornecer maior qualidade de vida para a população.

\section{REFERÊNCIAS}

1. Tavares W, Marinho LAC. Rotinas de diagnóstico e tratamento das doenças infecciosas e parasitárias. 4. ed. São Paulo: Atheneu, 2015.

2. Braga IA, Valle D. Aedes aegypti: histórico do controle no Brasil. Epidemiol. Serv. Saúde. Brasília [Internet], 2007 [citado 2020 Mai 17];6(2):113-118. doi: http://dx.doi.org/10.5123/S1679-49742007000200006

3. Defavari E, et al. Análise espacial da incidência da dengue em um município de médio porte do estado de São Paulo de 2008 a 2015. Rev. de Saúde Coletiva da UEFS, Feira de Santana [Internet], $2017 \quad$ [citado $2020 \quad$ Mai 17$] ; 7(3) 10-17 . \quad$ doi: $\underline{\text { http://dx.doi.org/10.13102/rscdauefs.v0i0.2560 }}$ 
4. Brasil. Ministério da Saúde. Secretaria de Vigilância à Saúde. Coordenação Geral de Informações e Análises Epidemiológicas. Sistema de Informações sobre Mortalidade, 2020. Disponível em: http://tabnet.datasus.gov.br/cgi/tabcgi.exe?sim/cnv/obt10uf.def

5. Brasil. Ministério da Saúde. Departamento de Ciência e Tecnologia. Secretaria de Ciência, Tecnologia e Insumos Estratégicos. Doenças negligenciadas: estratégias do Ministério da Saúde. Rev Saude Publica [Internet], 2010 [citado 2020 Mai 17];44(1):200-202. doi: https://doi.org/10.1590/S0034-89102010000100023

6. CID-10. Classificação Estatística Internacional de Doenças e Problemas Relacionados à Saúde. 10a rev. São Paulo: Universidade de São Paulo; 1997. vol.1. 5. Organização Mundial da Saúde.

7. Andrade JV; Moraes RCCM. O que o Coronavírus tem nos tirado? Anos potenciais de vida perdidos em Minas Gerais. J. nurs. health [Internet], 2020 [citado 2020 Set 16]; 10 (n.esp.):e20104014. doi: https://doi.org/10.15210/jonah.v10i4.19043

8. Brasil. Boletim Epidemiológico 2: Monitoramento dos casos de arboviroses urbanas transmitidas pelo Aedes aegypti (dengue, chikungunya e zika), Semanas Epidemiológicas 1 a 52, 2019. Brasília: Ministério da Saúde: Secretaria de Vigilância em Saúde. 2020.

9. Brasil. Boletim Epidemiológico 19: Monitoramento dos casos de arboviroses urbanas transmitidas pelo Aedes aegypti (dengue, chikungunya e zika), Semanas Epidemiológicas 1 a 18, 2020. Brasília: Ministério da Saúde, Secretaria de Vigilância em Saúde. 2020.

10. Teich V, et al. Aedes aegypti e sociedade: o impacto econômico das arboviroses no Brasil. J Bras Econ Saúde. Rio de Janeiro [Internet], 2017 [citado 2020 Mai 17];9(3): 267-276. Disponível em: https://pesquisa.bvsalud.org/portal/resource/pt/biblio-883013

11. Pepin KM, et al. Cost-effectiveness of novel system of mosquito surveillance and control, Brazil. Emerg Infect Dis. Atlanta [Internet], 2013 [citado 2020 Mai 17];19(4):542-50. doi: http://dx.doi.org/10.3201/eid1904.120117 
12. Ribeiro PC, et al. Perfil clínico-epidemiológico dos casos suspeitos de Dengue em um bairro da zona sul de Teresina, PI, Brasil. Rev. bras. enferm. [Internet]. 2008 [citado 2020 Mai 17];61(2):227-232. doi: https://doi.org/10.1590/S0034-71672008000200013

13. Ferreira AC, et al. Dengue em Araraquara, SP: epidemiologia, clima e infestação por Aedes aegypti. Rev. Saúde Pública [Internet]. 2018 [citado 2020 Mai 17]; 52: 18. doi: https://doi.org/10.11606/s1518-8787.2018052000414

14. Oliveira RM, Oliveira LRM. Epidemiologia da Dengue: análise em diversas regiões do Brasil. Rev. Cie. da Esc. de Saúde do Exército. Rio de Janeiro, [Internet]. 2019 [citado 2020 Mai 17];2(2):32-44. Disponível em: http://ebrevistas.eb.mil.br/index.php/RCEsSEx/article/view/2432

15. Silva JS, et al. A influência do clima urbano na proliferação do mosquito Aedes aegypti em Jataí (GO) na perspectiva da geografia médica. Hygeia. Uberlândia, [Internet]. 2007 [citado 2020 Mai 17];2(5):33-49, dez. 2007. Disponível em: http://www.seer.ufu.br/index.php/hygeia/article/view/16883 